\title{
Slackia equolifaciens sp. nov., a human intestinal bacterium capable of producing equol
}

\author{
Jong-Sik Jin, ${ }^{1,2,3}$ Maki Kitahara, ${ }^{3}$ Mitsuo Sakamoto, ${ }^{3}$ Masao Hattori ${ }^{2}$ \\ and Yoshimi Benno ${ }^{1}$ \\ ${ }^{1}$ Benno Laboratory, Center for Intellectual Property Strategies, RIKEN, 2-1 Hirosawa, Wako, \\ Saitama 351-0198, Japan \\ ${ }^{2}$ Institute of Natural Medicine, University of Toyama, 2630 Sugitani, Toyama 930-0194, Japan \\ ${ }^{3}$ Microbe Division/Japan Collection of Microorganisms, RIKEN BioResource Center, 2-1 Hirosawa, \\ Wako, Saitama 351-0198, Japan
}

Correspondence Jong-Sik Jin jin@riken.jp

\begin{abstract}
An equol-producing bacterium, strain $\mathrm{DZE}^{\top}$, which was isolated from human faeces, was characterized by morphological, biochemical and molecular methods. The isolate was Grampositive, obligately anaerobic, non-spore-forming, asaccharolytic and rod-shaped. 16S rRNA gene sequence analysis showed 92.8, 91.0, 91.1 and 90.6 \% similarities with Slackia faecicanis, Slackia exigua, Slackia heliotrinireducens and Slackia isoflavoniconvertens, respectively. Based on these data, we propose a novel species of the genus Slackia, Slackia equolifaciens sp. nov. The major cellular fatty acids are $\mathrm{C}_{14: 0}, \mathrm{C}_{18: 1} \omega 9 c$ and $\mathrm{C}_{18: 1} \omega 9 c$ DMA (dimethyl acetal). The DNA G $+C$ content of the strain is $60.8 \mathrm{~mol} \%$. The type strain of $S$. equolifaciens sp. nov. is DZE $\left(=\mathrm{JCM} 16059^{\top}=\right.$ CCUG $\left.58231^{\top}\right)$.
\end{abstract}

Dietary isoflavones such as puerarin, daidzin and daidzein have recently aroused interest because of their potential health benefits (Anderson et al., 1999; Kurzer \& Xu, 1997; Barnes, 1998; Setchell, 1998; Magee \& Rowland, 2004). After ingestion from edible plants, these three compounds are converted to $O$-desmethylangolensin and equol as end products (Supplementary Fig. S1, available in IJSEM Online) (Heinonen et al., 1999, 2003). Equol, in particular, has been extensively studied as an effective phytoestrogen. However, only $30-50 \%$ of individuals in the general population are capable of producing equol from daidzein (Rowland et al., 2000; Kelly et al., 1995). Because equol is produced exclusively by the intestinal bacterial metabolism of isoflavones, isolation and characterization of equolproducing bacterial strains has been attempted. Several strains have been reported to date (Maruo et al., 2008; Minamida et al., 2008).

The genus Slackia belongs to the family Coriobacteriaceae and its members are Gram-positive, non-motile, obligate anaerobes (Wade et al., 1999). Four species, Slackia faecicanis (Lawson et al., 2005), Slackia exigua, Slackia heliotrinireducens (Wade et al., 1999) and Slackia isoflavoniconvertens

The GenBank/EMBL/DDBJ accession number for the 16S rRNA gene sequence of strain DZE ${ }^{\top}$ is EU377663.

The proposed metabolic pathway of puerarin and daidzin by human intestinal bacteria, and cellular fatty acid compositions of strain DZE ${ }^{\top}$ and the type strains of closely related species are available with the online version of this paper.
(Matthies et al., 2009), have been described. S. isoflavoniconvertens, which was isolated from human faeces, is able to transform daidzein to equol.

We isolated a strain capable of transforming daidzein to equol from the faeces of a healthy female and reported its metabolic characteristics (Jin et al., 2008). A bacterial suspension from fresh faeces was repeatedly cultured in $2 \mathrm{ml}$ of general anaerobic medium (GAM) broth (Nissui) containing $0.1 \mathrm{mM}$ daidzein as a substrate at $37{ }^{\circ} \mathrm{C}$ in an anaerobic incubator. A portion of the culture, possessing metabolic activity, was seeded on GAM agar plates and incubated anaerobically for $72 \mathrm{~h}$ at $37{ }^{\circ} \mathrm{C}$. Colonies were repeatedly screened for their activity of transforming daidzein, to obtain a single daidzein-metabolizing bacterium (strain $\mathrm{DZE}^{\mathrm{T}}$ ). The strains used in the present study were maintained on GAM agar incubated for 3 days at $37{ }^{\circ} \mathrm{C}$ in an anaerobic jar (Hirayama) filled with $\mathrm{CO}_{2}$. Physiological and biochemical reactions were determined with an API 20A anaerobe test kit and a Rapid ID 32A anaerobe identification kit, respectively, as recommended by the manufacturer (bioMérieux). All tests were performed in duplicate. The DNA G+C content was determined by using the HPLC method of Tamaoka \& Komagata (1984). The elution solvent was a mixture of $0.02 \mathrm{M}\left(\mathrm{NH}_{4}\right) \mathrm{H}_{2} \mathrm{PO}_{4}$ and acetonitrile $(20: 1, \mathrm{v} / \mathrm{v})$. Fatty acid methyl esters were obtained from approximately $40 \mathrm{mg}$ of wet cells by saponification, methylation and extraction using minor modifications (Kuykendall et al., 1988) of the method of Miller (1982). Cells were cultured 
anaerobically on GAM agar ( $0.5 \%$ arginine-hydrochloride) for $96 \mathrm{~h}$ at $37{ }^{\circ} \mathrm{C}$. The $16 \mathrm{~S}$ rRNA gene sequence was analysed as described previously (Sakamoto et al., 2002). Almost 1500 bases of 16S rRNA gene sequence for strain $\mathrm{DZE}^{\mathrm{T}}$ were amplified by PCR with universal primers 27F (5'-AGAGTTTGATCCTGGCTCAG-3') and 1492R (5'-GGTTACCTTGTTACGACTT- $3^{\prime}$ ) using a Biometra Thermocycler Tgradient (Biometra). PCR products were purified by using an Ultraclean PCR Clean-up kit (MO $\mathrm{BIO}$ ) and were sequenced by using a BigDye Terminator cycle sequencing kit (Applied Biosystems) and ABI PRISM 3130 Genetic Analyzer (Applied Biosystems). Phylogenetic relatives of the bacterium were determined by performing database searches, and sequences of related species were retrieved from the DDBJ, EMBL and GenBank nucleotide sequence databases. Sequences were aligned by using CLUSTAL x (version 2.0) (Thompson et al., 1997) and a phylogenetic tree was reconstructed according to the neighbour-joining method (Saitou \& Nei, 1987). Minimum-evolution and maximum-parsimony phylogenetic trees were inferred by using the software package MEGA version 4.0 (Tamura et al., 2007). Equol production was examined by incubation of the bacteria with daidzein. GAM broth $(2 \mathrm{ml}$ ) containing daidzein (final concentration of $0.1 \mathrm{mM}$ ) was incubated with a bacterial suspension $(100 \mu \mathrm{l})$ at $37^{\circ} \mathrm{C}$ for $48 \mathrm{~h}$ under anaerobic conditions. A $100 \mu \mathrm{l}$ aliquot was taken out, extracted with $30 \mu \mathrm{l}$ of ethyl acetate and subjected to TLC, which was carried out on silica gel pre-coated $60 \mathrm{~F}_{254}$ plates $(0.25 \mathrm{~mm}$, Merck). The products were detected under a UV lamp or by exposure to $\mathrm{I}_{2}$ vapour.
Analysis of $16 \mathrm{~S}$ rRNA gene sequences revealed that the novel strain was a member of the family Coriobacteriaceae. Strain $\mathrm{DZE}^{\mathrm{T}}$ displayed highest sequence similarity with species of the genus Slackia [S. faecicanis (92.8\%), S. exigua $(91.0 \%)$, S. heliotrinireducens $(91.1 \%)$ and S. isoflavoniconvertens (90.6\%)] (Fig. 1). However, the strain exhibited substantially lower similarities with other members of the family Coriobacteriaceae [species of the genus Eggerthella (89.1-89.7\%), Denitrobacterium detoxificans (88.8\%) and Asaccharobacter celatus (91.1\%)]. Moreover, strain $\mathrm{DZE}^{\mathrm{T}}$ showed $91.4 \%$ similarity with Adlercreutzia equolifaciens, which also converts daidzein to equol. Although it is not possible to distinguish species on the basis of $16 \mathrm{~S}$ rRNA sequence similarities alone, it is clear that the greater than $7 \%$ gene sequence divergence observed between strain $\mathrm{DZE}^{\mathrm{T}}$ and the four currently recognized species of the genus Slackia is consistent with separate species status. It is now generally accepted that organisms showing $3 \%$ or greater 16S rRNA gene sequence divergence are not members of the same species (Lawson et al., 2005). Physiological and biochemical reactions of strain $\mathrm{DZE}^{\mathrm{T}}$ were studied with S. faecicanis JCM $14555^{\mathrm{T}}$ and S. exigua JCM $11022^{\mathrm{T}}$ as references. The phenotypic and biochemical characteristics that are useful in differentiating strain $\mathrm{DZE}^{\mathrm{T}}$ from other members of the genus Slackia are shown in Table 1. The characteristics of strain $\mathrm{DZE}^{\mathrm{T}}$ were similar to those of $S$. exigua. However, the two reference strains, $S$. faecicanis $\mathrm{JCM} 14555^{\mathrm{T}}$ and S. exigua JCM $11022^{\mathrm{T}}$, did not produce equol from daidzein. The long-chain cellular fatty acids of strain $\mathrm{DZE}^{\mathrm{T}}$ included $\mathrm{C}_{12: 0} \quad(2.9 \%)$, $\mathrm{C}_{14: 0}(15.1 \%), \mathrm{C}_{16: 0}(2.7 \%)$ and $\mathrm{C}_{18: 1} \omega 9 c(29.5 \%)$

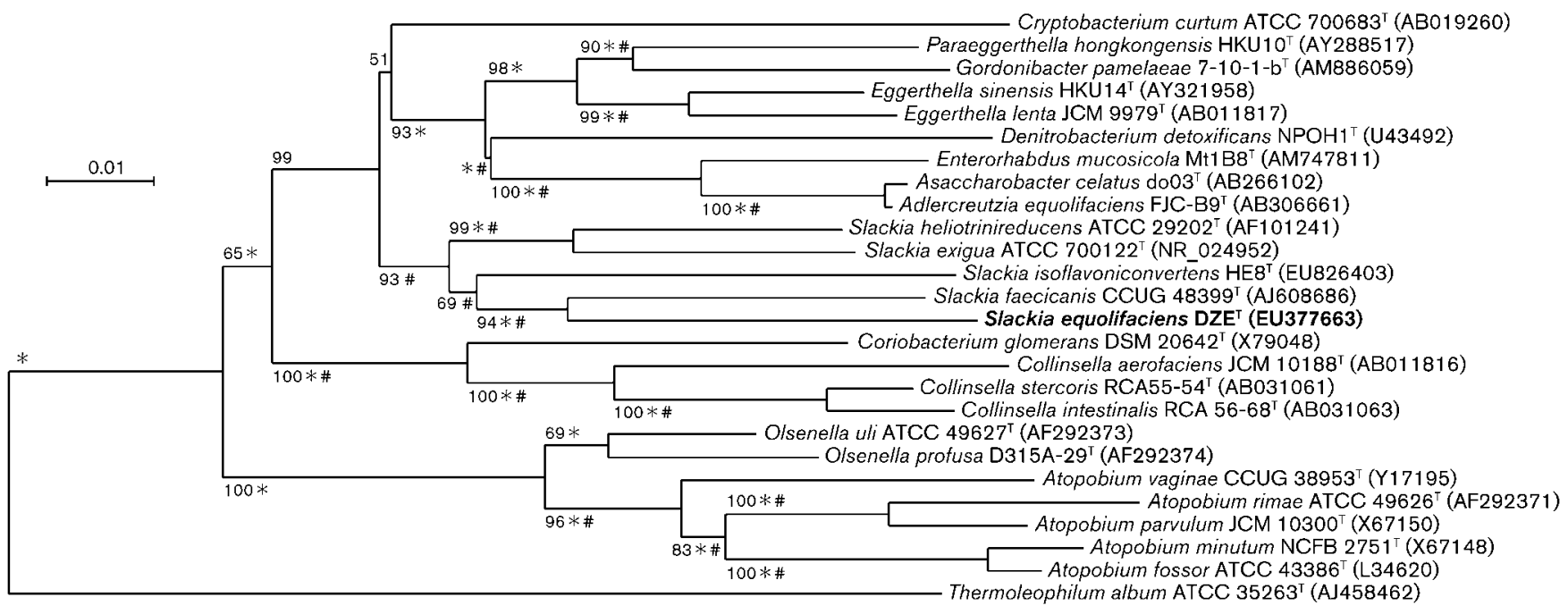

Fig. 1. Phylogenetic tree showing the relationship between strain $D Z E^{\top}$ and the type strains of related species. The tree was reconstructed by the neighbour-joining method based on 16S rRNA gene sequences (approx. 1320 nucleotides). Numbers at nodes indicate percentage bootstrap values of 1000 replicates. Bootstrap values above $50 \%$ are given at the branching points. Asterisks and hashes, respectively, indicate branches that were also recovered using the minimum-evolution and maximumparsimony methods. Bar, 0.01 substitutions per nucleotide position. Accession numbers for $16 \mathrm{~S}$ rRNA gene sequences are given for each strain. 
Table 1. Characteristics of strain $\mathrm{DZE}^{\top}$ and other members of the genus Slackia

Strains: 1, S. equolifaciens sp. nov. DZE ${ }^{\mathrm{T}}$; 2, S. exigua JCM $11022^{\mathrm{T}}$; 3, S. faecicanis JCM $14555^{\mathrm{T}}$; 4, S. heliotrinireducens JCM $14554^{\mathrm{T}}$; 5 , S. isoflavoniconvertens DSM 22006 ${ }^{\mathrm{T}}$. +, Positive; -, negative; ND, not detected; NT, not tested; v, variable. Data for S. heliotrinireducens and S. isoflavoniconvertens from Lawson et al. (2005), Wade et al. (1999) and Matthies et al. (2009).

\begin{tabular}{|c|c|c|c|c|c|}
\hline Characteristic & 1 & 2 & 3 & 4 & 5 \\
\hline Size $(\mu \mathrm{m})$ & $0.5 \times 0.8-1.0$ & $0.5 \times 1.0$ & $0.5 \times 1-2$ & $0.8 \times 0.8-1.2$ & $0.4 \times 2.4$ \\
\hline Nitrate reduction & - & - & $-^{*}$ & + & - \\
\hline Fermentation products from glucose & ND & ND & $\mathrm{ND}$ & Acetate & ND \\
\hline \multicolumn{6}{|l|}{ Enzyme activity } \\
\hline Proline arylamidase & + & + & - & + & - \\
\hline Leucyl glycine arylamidase & $\mathrm{V}$ & - & - & - & - \\
\hline Phenylalanine arylamidase & + & + & - & + & - \\
\hline Leucine arylamidase & + & + & - & + & - \\
\hline Tyrosine arylamidase & + & + & - & + & - \\
\hline Alanine arylamidase & + & + & - & + & - \\
\hline
\end{tabular}

${ }^{\star}$ Data from this study were different from published data (Lawson et al., 2005; Wade et al., 1999). These characteristics are assumed to be variable.

(Supplementary Table S1). Based upon phylogenetic and phenotypic findings, we propose the name Slackia equolifaciens sp. nov. for this strain.

\section{Description of Slackia equolifaciens sp. nov.}

Slackia equolifaciens (e.quo.li.fa'ci.ens. N.L. n. equol-olis equol; L. part. adj. faciens making; N.L. part. adj. equolifaciens equol-producing).

Cells are Gram-positive, rod-shaped $(0.5 \times 0.8-1.0 \mu \mathrm{m})$, anaerobic, non-motile and non-spore-forming. Colonies on GAM agar $(0.5 \%$ arginine-hydrochloride) plates are 1$2 \mathrm{~mm}$ in diameter and a translucent grey colour after 4 days at $37{ }^{\circ} \mathrm{C}$. Growth is stimulated by arginine. Indole test is negative. Acid is not produced from L-arabinose, cellobiose, glucose, lactose, maltose, D-mannitol, D-mannose, melezitose, raffinose, L-rhamnose, salicin, D-sorbitol, sucrose, trehalose or $\mathrm{D}$-xylose. Positive reactions are obtained using Rapid ID 32A for arginine dihydrolase, proline arylamidase, phenylalanine arylamidase, leucine arylamidase, tyrosine arylamidase, alanine arylamidase, glycine arylamidase, histidine arylamidase and serine arylamidase. Negative results are obtained for nitrate reduction, catalase, urease, $\alpha$-galactosidase, $\beta$-galactosidase, $\beta$-galactosidase- 6 -phosphate, $\alpha$-glucosidase, $\beta$-glucosidase, $\alpha$-arabinosidase, $\beta$-glucuronidase, $N$-acetyl- $\beta$-glucosaminidase, glutamic acid decarboxylase, $\alpha$-fucosidase, arginine arylamidase, pyroglutamic acid arylamidase and glutamyl glutamic acid arylamidase. The leucyl glycine arylamidase reaction is variable. The major cellular fatty acids are $C_{14: 0}$, $\mathrm{C}_{18: 1} \omega 9 c$ and $\mathrm{C}_{18: 1} \omega 9 c$ DMA (dimethyl acetal). The DNA $\mathrm{G}+\mathrm{C}$ content of the type strain is $60.8 \mathrm{~mol} \%$.
The type strain is $\mathrm{DZE}^{\mathrm{T}}\left(=\mathrm{JCM} 16059^{\mathrm{T}}=\mathrm{CCUG} 58231^{\mathrm{T}}\right.$ ), which was isolated from the faeces of a healthy human.

\section{References}

Anderson, J. J., Anthony, M. S., Cline, J. M., Washburn, S. A. \& Garner, S. C. (1999). Health potential of soy isoflavones for menopausal women. Public Health Nutr 2, 489-504.

Barnes, S. (1998). Evolution of the health benefits of soy isoflavones. Proc Soc Exp Biol Med 217, 386-392.

Heinonen, S., Wähälä, K. \& Adlercreutz, H. (1999). Identification of isoflavone metabolites dihydrodaidzein, dihydrogenistein, 6'-OH-O$\mathrm{dma}$, and cis-4-OH-equol in human urine by gas chromatographymass spectroscopy using authentic reference compounds. Anal Biochem 274, 211-219.

Heinonen, S. M., Hoikkala, A., Wähälä, K. \& Adlercreutz, H. (2003). Metabolism of the soy isoflavones daidzein, genistein and glycitein in human subjects. Identification of new metabolites having an intact isoflavonoid skeleton. J Steroid Biochem Mol Biol 87, 285299.

Jin, J.-S., Nishihata, T., Kakiuchi, N. \& Hattori, M. (2008). Biotransformation of $C$-glucosylisoflavone puerarin to estrogenic (3S)-equol in co-culture of two human intestinal bacteria. Biol Pharm Bull 31, 1621-1625.

Kelly, G. E., Joannou, G. E., Reeder, A. Y., Nelson, C. \& Waring, M. A. (1995). The variable metabolic response to dietary isoflavones in humans. Proc Soc Exp Biol Med 208, 40-43.

Kurzer, M. S. \& Xu, X. (1997). Dietary phytoestrogens. Annu Rev Nutr 17, 353-381.

Kuykendall, L. D., Roy, M. A., O'Neill, J. J. \& Devine, T. E. (1988). Fatty acids, antibiotic resistance, and deoxyribonucleic acid homology groups of Bradyrhizobium japonicum. Int J Syst Bacteriol 38, 358361. 
Lawson, P. A., Greetham, H. L., Gibson, G. R., Giffard, C., Falsen, E. \& Collins, M. D. (2005). Slackia faecicanis sp. nov., isolated from canine faeces. Int J Syst Evol Microbiol 55, 1243-1246.

Magee, P. J. \& Rowland, I. R. (2004). Phyto-oestrogens, their mechanism of action: current evidence for a role in breast and prostate cancer. Br J Nutr 91, 513-531.

Maruo, T., Sakamoto, M., Ito, C., Toda, T. \& Benno, Y. (2008). Adlercreutzia equolifaciens gen. nov., sp. nov., an equol-producing bacterium isolated from human faeces, and emended description of the genus Eggerthella. Int J Syst Evol Microbiol 58, 1221-1227.

Matthies, A., Blaut, M. \& Braune, A. (2009). Isolation of a human intestinal bacterium capable of daidzein and genistein conversion. Appl Environ Microbiol 75, 1740-1744.

Miller, L. T. (1982). Single derivatization method for routine analysis of bacterial whole-cell fatty acid methyl esters, including hydroxy acids. J Clin Microbiol 16, 584-586.

Minamida, K., Ota, K., Nishimukai, M., Tanaka, M., Abe, A., Sone, T., Tomita, F., Hara, H. \& Asano, K. (2008). Asaccharobacter celatus gen. nov., sp. nov., isolated from rat caecum. Int J Syst Evol Microbiol 58, 1238-1240.

Rowland, I. R., Wiseman, H., Sanders, T. A., Adlercreutz, H. \& Bowey, E. A. (2000). Interindividual variation in metabolism of soy isoflavones and lignans: influence of habitual diet on equol production by the gut microflora. Nutr Cancer 36, 27-32.

Saitou, N. \& Nei, M. (1987). The neighbor-joining method: a new method for reconstructing phylogenetic trees. Mol Biol Evol 4, 406-425.
Sakamoto, M., Suzuki, M., Umeda, M., Ishikawa, I. \& Benno, Y. (2002). Reclassification of Bacteroides forsythus (Tanner et al. 1986) as Tannerella forsythensis corrig., gen. nov., comb. nov. Int J Syst Evol Microbiol 52, 841-849.

Setchell, K. D. (1998). Phytoestrogens: the biochemistry, physiology, and implications for human health of soy isoflavones. Am J Clin Nutr 68, 1333S-1346S.

Tamaoka, J. \& Komagata, K. (1984). Determination of DNA base composition by reversed-phase high-performance liquid chromatography. FEMS Microbiol Lett 25, 125-128.

Tamura, K., Dudley, J., Nei, M. \& Kumar, S. (2007). MEGA4: molecular evolutionary genetics analysis (MEGA) software version 4.0. Mol Biol Evol 24, 1596-1599.

Thompson, J. D., Gibson, T. J., Plewniak, F., Jeanmougin, F. \& Higgins, D. G. (1997). The CLUSTAL_X windows interface: flexible strategies for multiple sequence alignment aided by quality analysis tools. Nucleic Acids Res 25, 4876-4882.

Wade, W. G., Downes, J., Dymock, D., Hiom, S. J., Weightman, A. J., Dewhirst, F. E., Paster, B. J., Tzellas, N. \& Coleman, B. (1999). The family Coriobacteriaceae: reclassification of Eubacterium exiguum (Poco et al. 1996) and Peptostreptococcus heliotrinreducens (Lanigan 1976) as Slackia exigua gen. nov., comb. nov. and Slackia heliotrinireducens gen. nov., comb. nov., and Eubacterium lentum (Prevot 1938) as Eggerthella lenta gen. nov., comb. nov. Int J Syst Bacteriol 49, 595-600. 